\title{
Photonic Time-Stretch Spectroscopy for Multiplex Stimulated Raman Scattering
}

\author{
Francesco Saltarelli ${ }^{1}$, Vikas Kumar $^{1}$, Daniele Viola ${ }^{1}$, Francesco Crisafi $^{1}$, Fabrizio Preda ${ }^{1}$, \\ Giulio Cerullo ${ }^{1}$ and Dario Polli ${ }^{1,2, *}$ \\ ${ }^{1}$ CNR-IFN and Dipartimento di Fisica, Politecnico di Milano, P.za L. da Vinci 32, 20133 Milano, \\ Italy \\ ${ }^{2}$ Center for Nano Science and Technology@PoliMi, Istituto Italiano di Tecnologia, Via Giovanni \\ Pascoli 70/3, 20133 Milano, Italy
}

\begin{abstract}
Stimulated Raman scattering spectroscopy enables label-free molecular identification, but its broadband implementation is technically challenging. We experimentally demonstrate a novel approach to multiplex stimulated Raman scattering based on photonic time stretch. A telecom fiber stretches the broadband femtosecond Stokes pulse after the sample to $\approx 15 \mathrm{~ns}$, mapping its spectrum in time. The signal is sampled through a fast oscilloscope, providing single-shot spectra at $80-\mathrm{kHz}$ rate. We demonstrate high sensitivity in detecting the Raman vibrational modes of various samples over the entire high-frequency $\mathrm{C}-\mathrm{H}$ stretching region. Our results pave the way to high-speed broadband vibrational imaging for materials science and biophotonics.
\end{abstract}

\section{Introduction: state of the art of broadband SRS}

Raman spectroscopy allows for label-free identification of molecules, retrieving their characteristic vibrational fingerprints. It finds applications in many disciplines, such as biomedical sciences [1]. The cross section of spontaneous Raman scattering is inherently very low, thus hampering its use for high-speed microscopy applications. Coherent Raman scattering (CRS) boosts the signal by many orders of magnitude, allowing threedimensional imaging up to the video rate [2]. Stimulated Raman Scattering (SRS) is a CRS technique requiring two ultrashort laser pulses (called pump and Stokes). In its standard implementation, it probes a single vibrational line, thus providing limited information on the target's chemical composition. Combining a narrowband (picosecond) pump with a broadband (femtosecond) Stokes, on the other hand, it is possible to retrieve the full Raman spectrum at once. In this configuration, called broadband or multiplex SRS, however, it is technically challenging to push the acquisition speed below a millisecond per pixel (or voxel). Therefore, in recent years, there has been a great effort in speeding up the acquisition time of multiplex SRS [3].

\footnotetext{
*Corresponding author: dario.polli@polimi.it
} 


\section{Working principle of Photonic Time-Stretch SRS}

In this paper, we illustrate a novel approach to multiplex SRS, based on Photonic Time Stretch (PTS) [4]. This technique can acquire single-shot optical spectra at up to tens of $\mathrm{MHz}$ repetition rates by a high-speed analog-to-digital conversion [5]. The working principle is illustrated in Fig. 1. The simulations (based on numerical solving of the Nonlinear Schrodinger Equation) consider a $\approx 300$-fs pulse (see Fig. 1(a)) with spectrum presenting some sharp features (see Fig. 1(b)) due to stimulated Raman gain. The amplitude of these features is macroscopic for sake of clarity; in the real case, it will be 3-4 orders of magnitude lower. After propagation in a long standard optical fiber, the temporal profile of the pulse (see Fig. 1(c)) perfectly maps its spectrum (see Fig. 1(d)). Care is taken to avoid non-linearities in the fiber, which would otherwise distort the output spectrum.

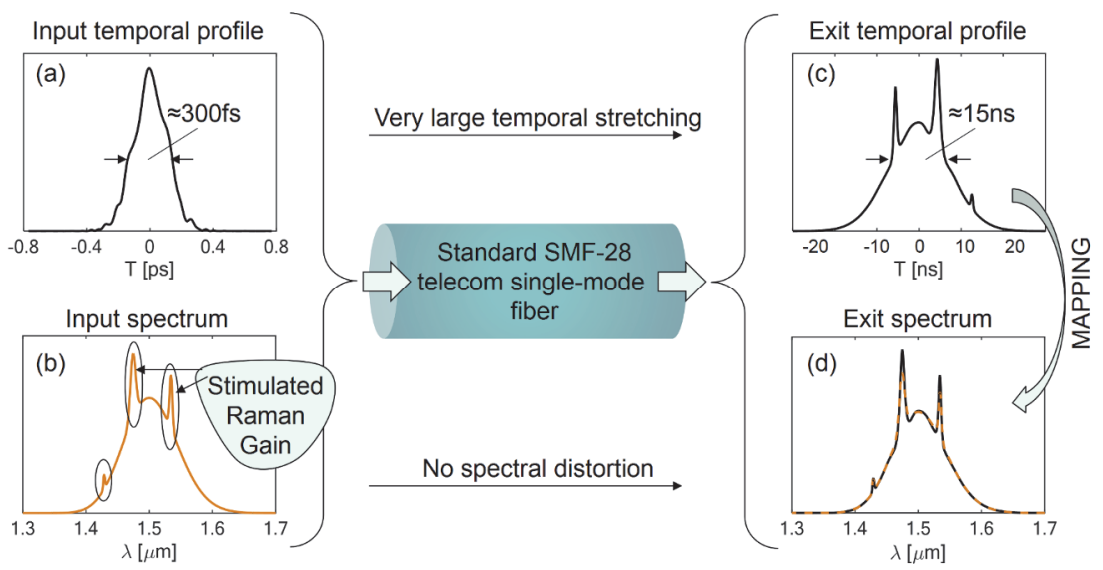

Fig. 1. Working principle of photonic time stretching.

\section{Experimental demonstration of PTS-SRS}

Figure 2 depicts our PTS-SRS experimental setup. A narrowband pump (at $1.03 \mu \mathrm{m}$ ) and a broadband Stokes generated by a collinear optical parametric amplifier (OPA) (at $1.50 \mu \mathrm{m}$, with $50-\mathrm{nm}$ bandwidth) are focused on the sample to generate the Raman signal. After the sample, a grating pre-stretcher and an $18.6 \mathrm{~km}$-long single-mode fiber disperse the Stokes pulse to a final duration of a few tens of nanoseconds. A fast photodiode and a high-speed oscilloscope finally acquire its temporal profile, which is uniquely mapped into a spectrum by simply calibrating the introduced dispersion.

Our system, based on an $80-\mathrm{kHz}$ repetition-rate laser, covers a Raman spectral window of $500 \mathrm{~cm}^{-1}$ with a resolution of $10 \mathrm{~cm}^{-1}$. We compared an oscilloscope with $1 \mathrm{GHz}$ bandwidth but 12-bit resolution with another having $2.5 \mathrm{GHz}$ bandwidth and 8-bit resolution. The former enabled higher sensitivity, reaching $10^{-3}$ in $400-\mu$ s measurement time and approaching $2 \cdot 10^{-5}$ for 1 -s integration (see Fig. 3(a)). The latter, on the other hand, allowed for a higher spectral resolution but it provided a lower sensitivity. Fig. 3(b) plots representative SRS spectra collected in different solvents. Figure 3(c) reports the results of a dilution test of methanol in water, showing that the system can detect methanol down to a concentration of a few percent. 


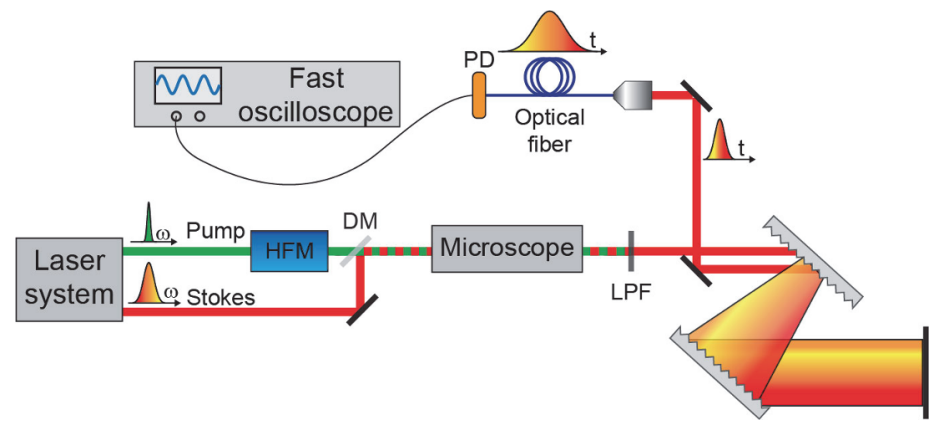

Fig. 2. Experimental setup of the PTS-SRS. HFM: high-frequency modulator; DM: dichroic mirror; LPF: long-pass filter; PD: photodiode.
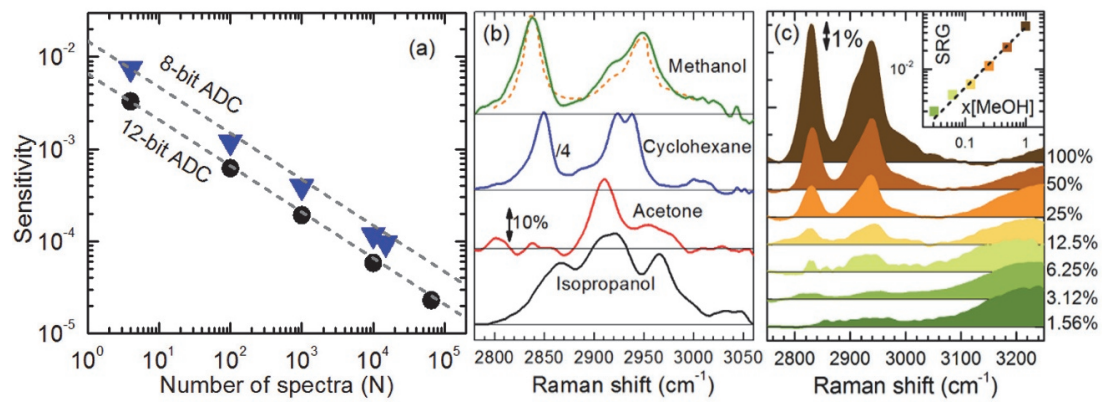

Fig. 3 (a) Sensitivity of the PTS-SRS: rms of the SRG as a function of $\mathrm{N}$ for the 8-bit (blue triangles) and the 12-bit (black circles) oscilloscopes. Dashed lines are fits to the data with $\sqrt{ } \mathrm{N}$ functions. (b) SRS spectra of different solvents, acquired in $100 \mu$ s. Orange dashed line is a reference spontaneous Raman spectrum of methanol. Pump energy was 200nJ on the sample. (c) Dilution test of methanol in water. Inset: $\mathrm{SRG}$ of the $2840 \mathrm{~cm}^{-1}$ peak as a function of methanol concentration.

These performances are already suitable for a variety of applications, such as monitoring microfluidic flows or the onset of chemical reactions [6]. We anticipate two major improvements of our technique: (a) as the acquisition speed of this method does not depend on the acquired spectral region, we could extend the spectral coverage by simply using a broader Stokes pulse; (b) we could considerably shorten the acquisition time in the near future using a laser with $\mathrm{MHz}$ repetition rate. These results pave the way to an extension of high-speed coherent Raman imaging of samples to their entire vibrational signature.

\section{References}

[1] M. Ghomi, Applications of Raman spectroscopy to biology (IOS, 2012).

[2] B. G. Saar, C.W. Freudiger, J. Reichman, C.M. Stanley, G.R. Holtom, X.S. Xie, Science 330, 1368 (2010).

[3] C. H. Camp Jr. and M. T. Cicerone, Nat. Photon. 9, 295 (2015).

[4] F. Saltarelli, V. Kumar, D. Viola, F. Crisafi, F. Preda, G. Cerullo, and D. Polli, Opt. Expr. 24, 21264 (2016).

[5] A.M. Fard, S. Gupta, and B. Jalali, Laser Photonics Rev. 7, 207 (2013).

[6] G. V. Oshovsky, G. Rago, J. P. R. Day, M. L. Soudijn, W. Rock, S. H. Parekh, G.

Ciancaleoni, J. N. H. Reek and M. Bonn, Anal. Chem. 85, 8923 (2013). 